\title{
Early reduction in PD-L1 expression predicts faster treatment response in human cutaneous leishmaniasis
}

\author{
Nidhi S. Dey, ${ }^{1}$ Sujai Senaratne, ${ }^{2}$ Vijani Somaratne, ${ }^{3}$ Nayani P. Madarasinghe, ${ }^{4}$ Bimalka Seneviratne,,${ }^{5}$ Sarah Forrester, ${ }^{1}$ \\ Marcela Montes de Oca, ${ }^{1}$ Luiza Campos Reis, ${ }^{6}$ Srija Moulik, ${ }^{7}$ Pegine B. Walrad, ${ }^{8}$ Mitali Chatterjee, ${ }^{7}$ Hiro Goto, ${ }^{6,9}$ \\ Renu Wickremasinghe, ${ }^{2}$ Dimitris Lagos, ${ }^{1}$ Paul M. Kaye, ${ }^{1}$ and Shalindra Ranasinghe ${ }^{2}$ \\ ${ }^{1}$ York Biomedical Research Institute, Hull York Medical School, University of York, York, United Kingdom. ²Department of Parasitology, University of Sri Jayewardenepura, Nugegoda, Sri Lanka. \\ ${ }^{3}$ Dermatology Unit, District General Hospital Embilipitiya, Embilipitiya, Sri Lanka. ${ }^{4}$ Dermatology Unit, Teaching Hospital Anuradhapura, Anuradhapura, Sri Lanka. ${ }^{5}$ Deparment of Pathology, University of \\ Sri Jayewardenepura, Nugegoda, Sri Lanka. ${ }^{6}$ nstituto de Medicina Tropical de São Paulo, Faculdade de Medicina, Universidade de Sao Paulo, Sao Paulo, Brazil. ’Department of Pharmacology, Institute of \\ Postgraduate Medical Education and Research, Kolkata, India. ${ }^{8}$ York Biomedical Research Institute, Department of Biology, University of York, York, United Kingdom. ${ }^{9}$ Departamento de Medicina Preventiva, \\ Faculdade de Medicina, Universidade de São Paulo, São Paulo, Brazil.
}

\begin{abstract}
Cutaneous leishmaniasis (CL) is caused by Leishmania donovani in Sri Lanka. Pentavalent antimonials (e.g., sodium stibogluconate [SSC]) remain first-line drugs for CL with no new effective treatments emerging. We studied whole blood and lesion transcriptomes from Sri Lankan patients with CL at presentation and during SSC treatment. From lesions but not whole blood, we identified differential expression of immune-related genes, including immune checkpoint molecules, after onset of treatment. Using spatial profiling and RNA-FISH, we confirmed reduced expression of programmed death-ligand 1 (PD-L1) and indoleamine 2,3-dioxygenase 1 (IDO1) proteins on treatment in lesions of a second validation cohort and further demonstrated significantly higher expression of these checkpoint molecules on parasite-infected compared with noninfected lesional CD68 monocytes and macrophages. Crucially, early reduction in PD-L1 but not ID01 expression was predictive of rate of clinical cure $(H R=4.88)$ and occurred in parallel with reduction in parasite load. Our data support a model whereby the initial antileishmanial activity of antimonial drugs alleviates checkpoint inhibition on $\mathrm{T}$ cells, facilitating immune-drug synergism and clinical cure. Our findings demonstrate that PD-L1 expression can be used as a predictor of rapidity of clinical response to SSC treatment in Sri Lanka and support further evaluation of PD-L1 as a host-directed therapeutic in leishmaniasis.
\end{abstract}

\section{Introduction}

Every year, approximately 600,000 to 1 million new cases of cutaneous leishmaniasis (CL) occur, with a broad global distribution, often leading to stigma and reduced life chances and placing a burden on health services (1-3). Treatment options for CL have changed little in the more than 70 years since pentavalent antimonial drugs were first introduced, and there are limited new treatments on the horizon (3). Sri Lanka is endemic for CL, with the first autochthonous case being reported in 1992 (4). Sri Lankan CL is caused by Leishmania donovani zymodeme MON-37 parasite (5-7), usually associated with visceral leishmaniasis in other endemic countries. Current treatment for CL in Sri Lanka involves weekly intralesional or daily intramuscular administration of sodium stibogluconate (SSG), with or without cryotherapy, based on the site and size of the lesion and response to treatment. Cure often takes many months, and some patients may fail to respond completely or withdraw from treatment (8).

Authorship note: NSD and SS contributed equally to this work and are co-first authors. Conflict of interest: The authors have declared that no conflict of interest exists. Copyright: $\odot 2021$, Dey et al. This is an open access article published under the terms of the Creative Commons Attribution 4.0 International License.

Submitted: July 28, 2020; Accepted: September 28, 2021; Published: November 15, 2021. Reference information: J Clin Invest. 2021;131(22):e142765.

https://doi.org/10.1172/JCl142765.
Most of our understanding of the host immune response in CL stems from experimental models, and human disease is much less understood (9). Immune checkpoint molecules have been implicated in disease progression in preclinical models (10-17), but their role in human CL has not been explored. It is widely proposed that immune-drug synergy is required for effective treatment and that host-directed therapy (HDT) may have a future role in patient management (18-20), but few validated targets have emerged. Here, we searched for early correlates of treatment response that might be used to stratify patient response. Our results indicate an intimate relationship between intracellular parasitism and immune checkpoint molecule expression, with programmed death-ligand 1 (PD-L1) emerging as a promising target for HDT in Sri Lanka.

\section{Results and Discussion}

We first conducted a targeted transcriptomic analysis of the lesion site in a test cohort of 6 patients with typical homogeneous nodulo-ulcerative CL lesions ( 3 females, 3 males; mean age \pm SD, $34 \pm 11$ years; (Supplemental Figures 1-3 and Supplemental Table 1; supplemental material available online with this article; https://doi.org/10.1172/JCI142765DS1). Principal component analyses of lesion transcriptomic data showed separation of pre- and on-treatment samples in most patients (Figure 1A) and 120 differentially expressed genes (DEGs) were identified 
A

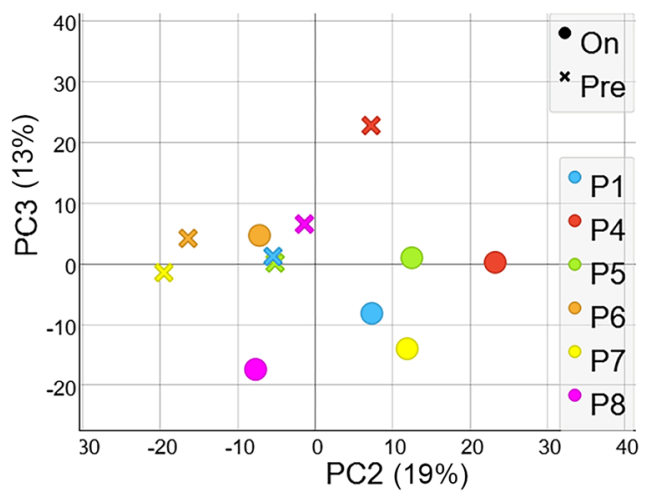

C

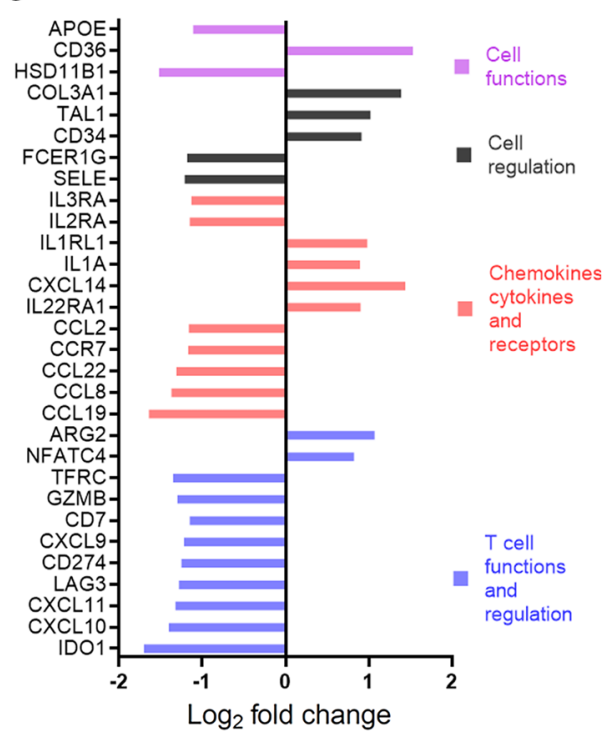

B

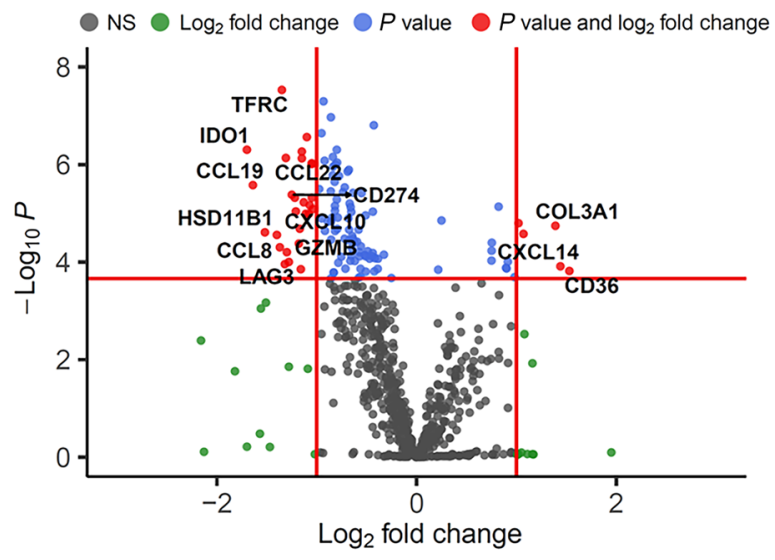

D

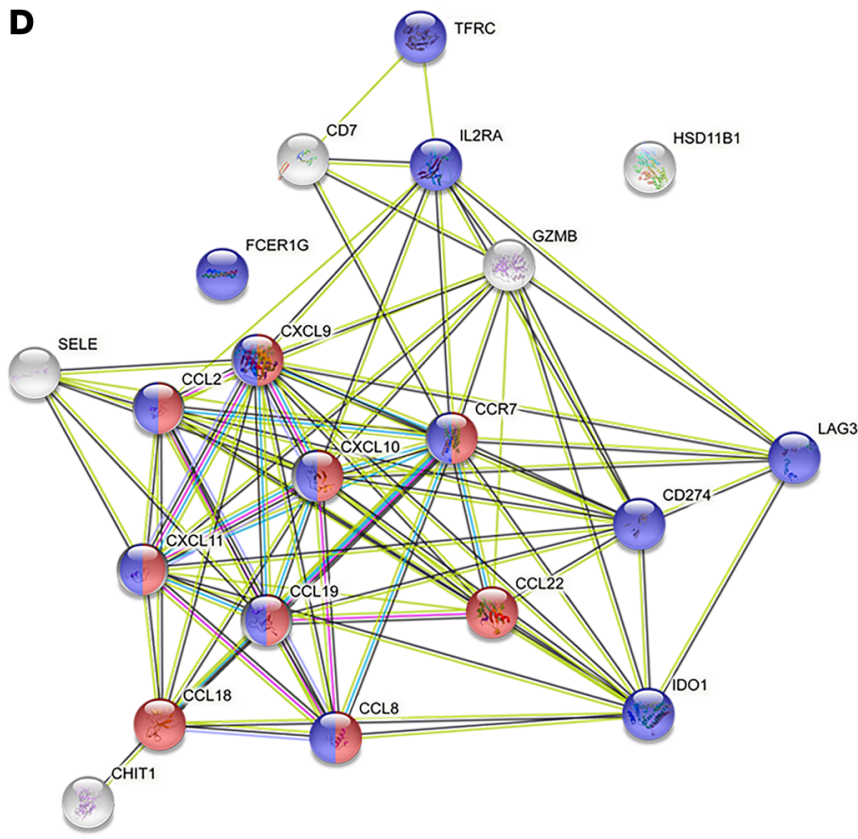

Figure 1. Differential expression and network analysis of genes regulated by drug treatment in lesions of Sri Lankan patients with CL. Immune-targeted tissue transcriptomics were conducted on tissue sections from test cohort patients comparing transcriptomes at presentation and on treatment. (A) Principal component analysis was performed to show differences between the pre- and on-treatment transcriptome of each patient based on 770 genes from the nCounter PanCancer Immunology Panel $(n=6)$. (B) DEGs comparing pretreatment biopsies with biopsies taken after 2 weeks on treatment (SSG). Cut off (red line) drawn at equivalent of adjusted $P=0.01$ and log (fold change) of 1 . (C) Top 30 genes that changed in expression on SSC treatment. (D) STRING protein-protein interaction network (https://string-db.org; ref. 22) analysis of genes listed in Supplemental Table 3 downregulated on SSG treatment. Pathways represent GO: 0072676, lymphocyte migration (red spheres), and G0: 0002684, positive regulation of immune system process (blue spheres). Top 20 genes are shown $\left(\log _{2}\right.$ fold change $\left.\geq 1.15\right)$ for clarity.

(FDR adjusted $P<0.01$; Figure 1B). In contrast, no DEGs were identified by RNA-seq in whole blood (Supplemental Figure 4 and Supplemental Table 2), suggesting that unlike CL caused by L. braziliensis (21), CL due to L. donovani in Sri Lanka is not accompanied by an overt systemic immune response.

Following treatment, the majority of DEGs in dermal lesions were downregulated $(87 \% ; 105 / 120)$, suggesting a reduction in inflammation following treatment (105 downregulated, 15 upregulated; Figure 1B and Supplemental Table 3). Genes for cellular functions and regulation, chemokines, membrane receptors, and $\mathrm{T}$ cell function and regulation were among the top 20 DEGs (Figure 1C). Further, STRING analysis (22) identified lymphocyte migration (Gene Ontology database [GO]: 0002687, FDR $=1.06 \times 10^{-14}$; includ- ing interferon inducible chemokines such as CXCL9, CXCL1O, CXCL11, CCL19, CCL8) and regulators of immune response (GO: 0002684, FDR $=1.94 \times 10^{-11}$; including IDO1, LAG3, and CD274/ PDL1) as highly enriched pathways (Figure 1D). Transcripts of inflammatory mediators including CXCL1O, GZMB, CCL2, and CCR7 (receptor for CCL19), previously shown to be associated with other forms of murine (23-25) or human (26-28) CL were also downregulated with initiation of treatment (Supplemental Table 3).

We next conducted multiplexed antibody digital spatial profiling (29) for 59 immune targets, selecting regions of interest (ROIs) based on expression of $\mathrm{CD}^{+}$and/or $\mathrm{CD} 68^{+}$(Supplemental Figure 5 and Figure 2, A-F). The t-SNE dimensional reduction on a total of 33 ROIs analyzed from 3 patients (P4, P6, and P7; Figure 2G) indicated 
A

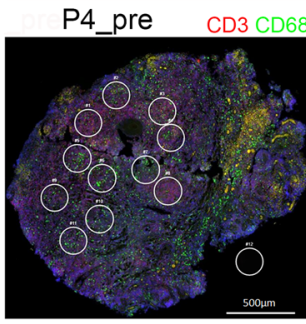

D

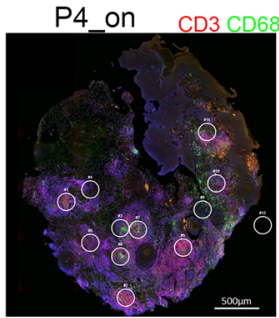

B

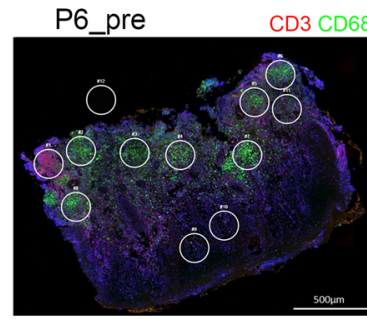

E

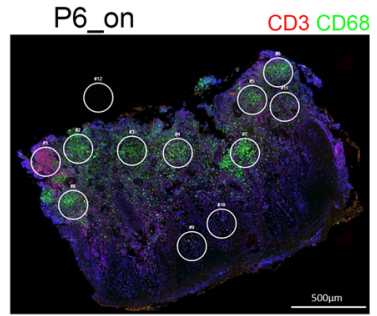

C P7 pre

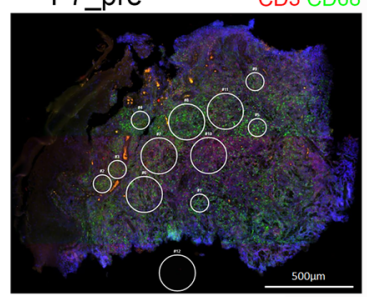

$\mathbf{F}$

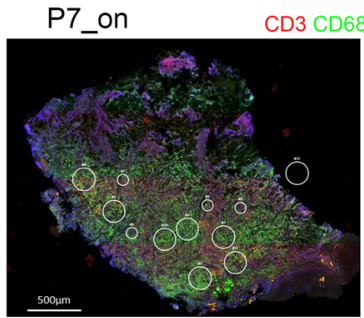

G
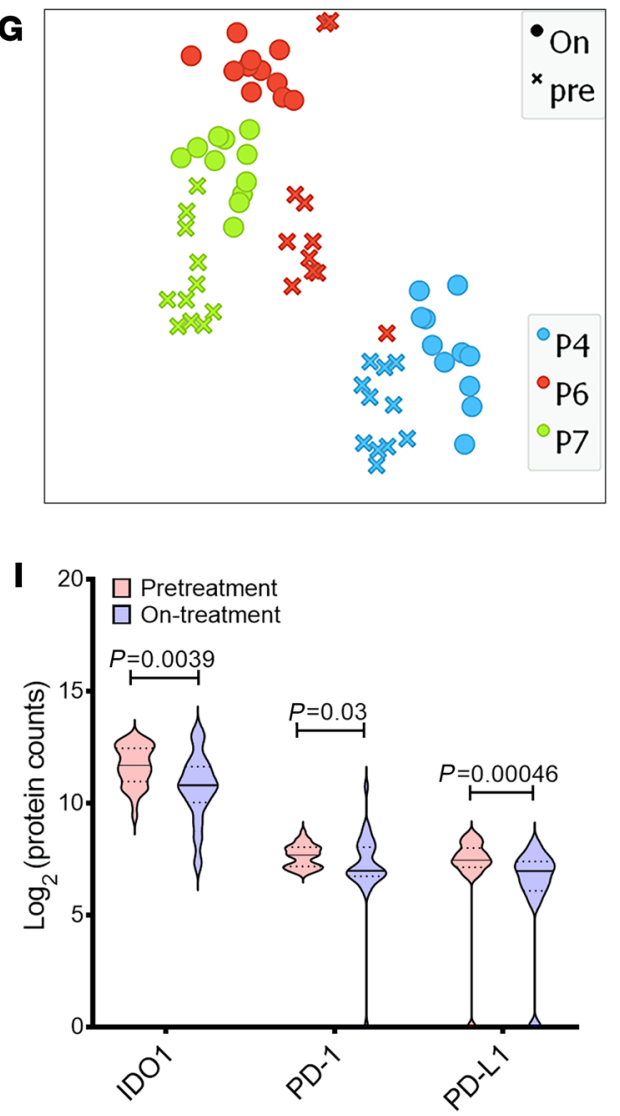

H NS $\bigcirc$ Log fold change $P$ value $P$ value and $\log _{2}$ fold change

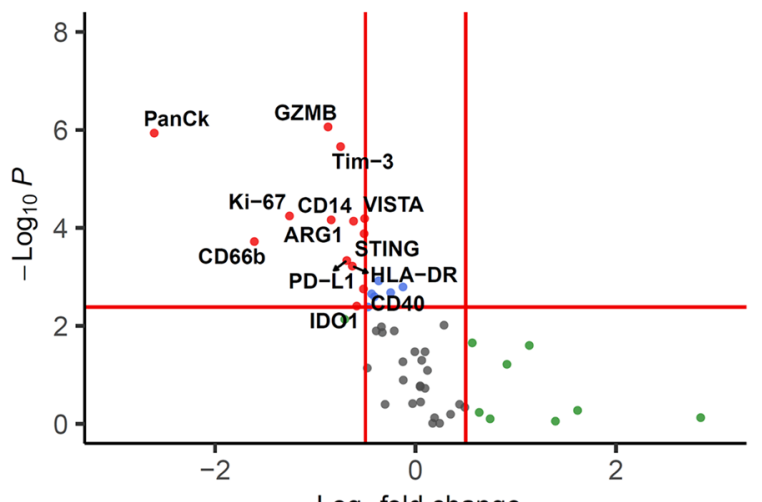

$\log _{2}$ fold change

J

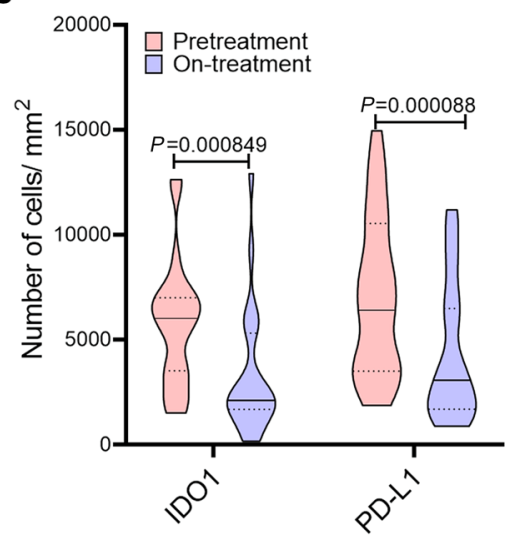

Figure 2. Digital spatial profiling of CL lesions. Digital spatial profiling was performed on tissue sections from test cohort individuals comparing ROIs from pre and on-treatment biopsies. (A-F) ROIs on $\mathrm{CD3}^{+}$and/or $\mathrm{CD68}^{+}$rich areas from pre- and on-treatment biopsies from patients $\mathrm{P4}$, $\mathrm{P6}$, and $\mathrm{P7}$ (CD68, green; CD3, red; Syto13, blue). Original magnification: $\times 20$; scale bar: $500 \mu \mathrm{m}$. (G) t-SNE plot based on 20 PCA loadings colored on patient ID. (H) Differential protein expression analysis comparing pretreatment to on-treatment ROls. Red lines indicate adjusted $P$ value cut off of $1 \%$ (Mann-Whitney test with FDR correction based on Benjamini, Krieger, and Yekutieli 2-stage set-up method) and log fold change $=0.5$ ( $n=33$ ROIs). (I) ID01, PD-1, and PD-L1 expression in pre- and on-treatment ROls. Mann Whitney rank test ( $n=33 \mathrm{ROI}$ ). (J) Immunohistochemistry (IHC) was performed on sections from patients in the validation cohort $(n=23)$ before and during treatment, and quantitated using StrataQuest (see Methods). Comparisons were by Wilcoxon matchedpairs signed rank test. Dotted lines show upper and lower quantile in I and J, median by solid line.

a considerable degree of interpatient heterogeneity in pretreatment lesional protein profiles, but with clear discrimination for each patient between pre- and on-treatment ROIs. Upon treatment, indoleamine 2,3-dioxygenase 1 (IDO1) and PD-L1 as well as PD-1 were selectively reduced in expression (Figure 2, $\mathrm{H}$ and I). STRING analysis of all discoveries based on FDR (5\%) also indicated significant enrichment in GO: 002684, as well as a pathway associated with regulation of $\mathrm{T}$ cell activation (GO: 0050863; Supplemental Figure 6, A and B). 
A

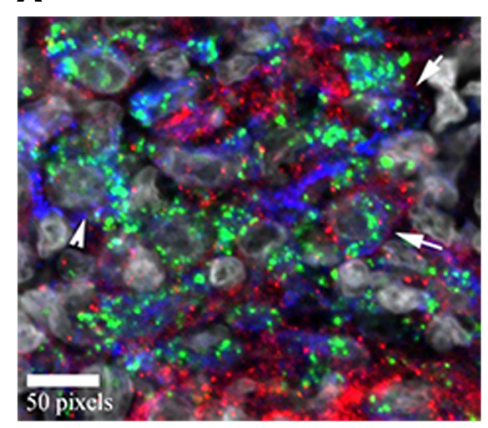

B

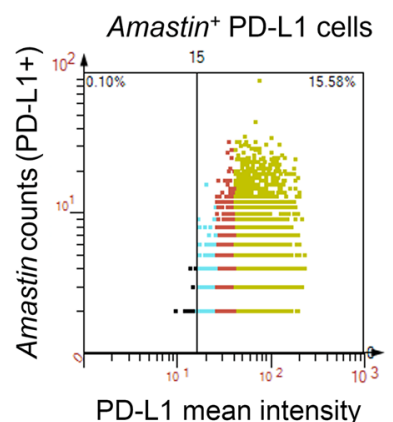

C

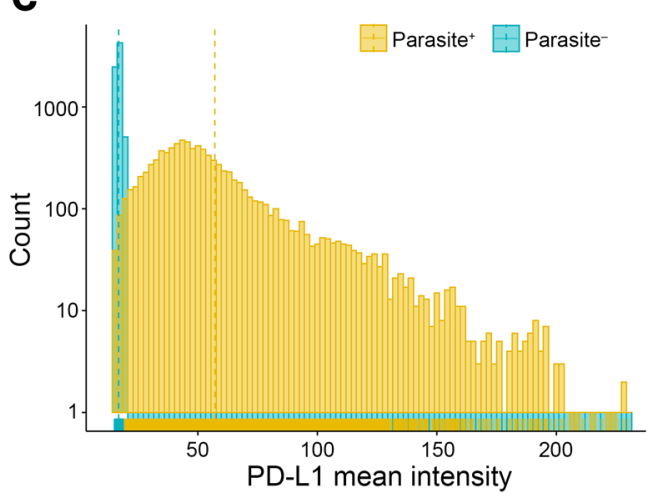

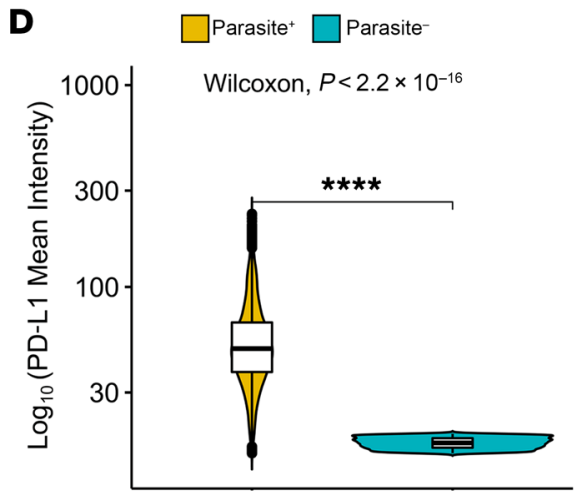

$\mathbf{E}$

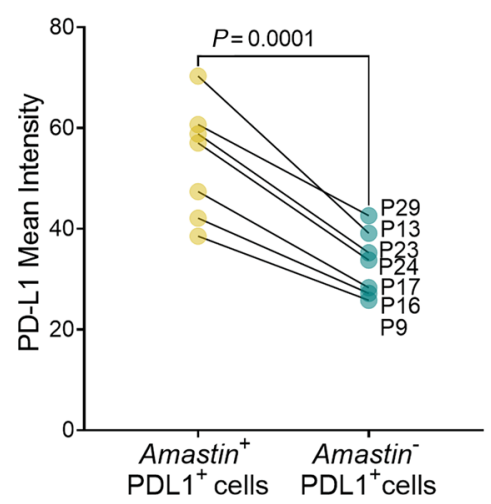

Figure 3. Immunofluorescence analyses of PD-L1 in infected and uninfected cells. Dual IHC-FISH using an Amastin probe was performed on pretreatment sections of patients enrolled in the validation cohort. (A) A 400 x confocal image showing infection of PD-L1+CD68 ${ }^{+}$(arrows) and PD-L1-CD68 ${ }^{+}$(arrowhead) cells. Scale bar: 50 pixels. (B) Relationship between PD-L1 expression and parasite burden (Amastin dot count). Scattergram from a representative patient (P24 at presentation) showing Amastin ${ }^{+}$low (cyan), medium (red), and high (green) PD-L1-expressing cells with respect to parasite abundance. (C) Fluorescence intensity distributions of infected and uninfected PD-L1 cells. (D) Mean fluorescence intensity of PD-L1 expression on Amastin' cells compared with Amastin $^{+}$cells from representative patient P24. The upper and lower whisker represents highest and lowest value that is within 1.5 times the interquartile range. $n=9159$ parasite positive cells and $n=41520$ parasite negative cells. Significance score was generated using Wilcoxon signed rank test. (E) PD-L1 expression on Amastin ${ }^{+} \mathrm{PD}-\mathrm{L}^{+}$cells versus Amastin-PD-L1+ cells ( $n=7$ patients). Significance score was generated using Student's 2 -tailed paired $t$ test after testing for normality using Shapiro-Wilk and Kolkogorov-Smirnov tests.

As IDO1 and PD-L1 have been targeted in cancer immunotherapy and hold promise for drug repurposing, we next sought to further validate these findings using quantitative IHC in an independent cohort of patients with CL ( 5 females, 18 males; mean age \pm SD, $44 \pm 11$ years; time to diagnosis, $7.76 \pm 8.2$ months; Supplemental Figures 7 and 8 and Supplemental Table 4) sampled at baseline and after 4 weeks of treatment. Using an accepted cut-off of greater than $5 \%$ of cells being positive (30), all patients $(n=23)$ expressed IDO1 (histochemical score [H-score] median $=81.2$; range $16-165$; ref. 31 ) and 20 of 23 patients had a reduction in the abundance of $\mathrm{IDO}^{+}$cells on treatment $(\mathrm{H}$-score median $=32$; range $1-171 ; P=0.0023$; Figure $2 \mathrm{~J})$. All patients were PD-L1 positive at presentation $(n=23 ; \mathrm{H}$-score median $=82.8$; range $12-164$ ) and 20 of 23 patients exhibited a reduction in the number of PD-L1-expressing cells on treatment (Figure 2J; H-score median $=36.7$; range $12.3-36.7 ; P=0.0008$ ). Collectively, these data indicate that IDO1 and PD-L1 are highly expressed in the lesions of Sri Lankan patients with CL and reduction in expression of these 2 checkpoint molecules represents an early response to SSG.

Though in vitro studies have indicated that intracellular parasitism by Leishmania could impact on the expression of immune checkpoint molecules (32-34), this has not been established in situ during human disease. To address this question, we combined IHC with RNA-FISH (35) to identify Amastin transcripts (as a surrogate for viable amastigotes) with a bespoke StrataQuest image analysis pipeline (Supplemental Figure 9, A-F). In 7 patients studied who were Amastin $^{+}$at presentation (Supplemental Methods), PD-L1 expression colocalized with $\mathrm{CD} 68^{+}$macrophages and parasitized cells were both PD-L1 ${ }^{+}$and PD-L1 ${ }^{-}$(Figure $3 \mathrm{~A}$ ). We binned the Amastin ${ }^{+} \mathrm{PDL}^{+}$and Amastin ${ }^{-} \mathrm{PDL1}^{+}$cells based on PD-L1 mean fluorescence intensity (Figure 3, B-D) and found that cells containing abundant Amastin transcripts expressed more PD-L1 than cells with fewer or no Amastin transcripts (Figure 3, B-E, Supplemental Figure 9, G-L, and Supplemental Figure 10). To independently corroborate this observation, we showed that a Sri Lankan strain of L. donovani was also capable of inducing upregulation of PD-L1 expression on human monocyte-derived macrophages in vitro (Supplemental Figure 11, A-F), as previously described for L. major (34). Similarly, IDO1 extensively colocalized with CD68 ${ }^{+}$ cells (Supplemental Figure 12A) and both $\mathrm{IDO}^{+} \mathrm{CD}^{+} 8^{+}$and $\mathrm{IDO}^{-}$ $\mathrm{CD} 8^{+}$cells were infected (Supplemental Figure 12B). Using a similar gating strategy (Supplemental Figure 12, C-H; $n=3$ patients), we found that cells with abundant Amastin transcripts expressed 

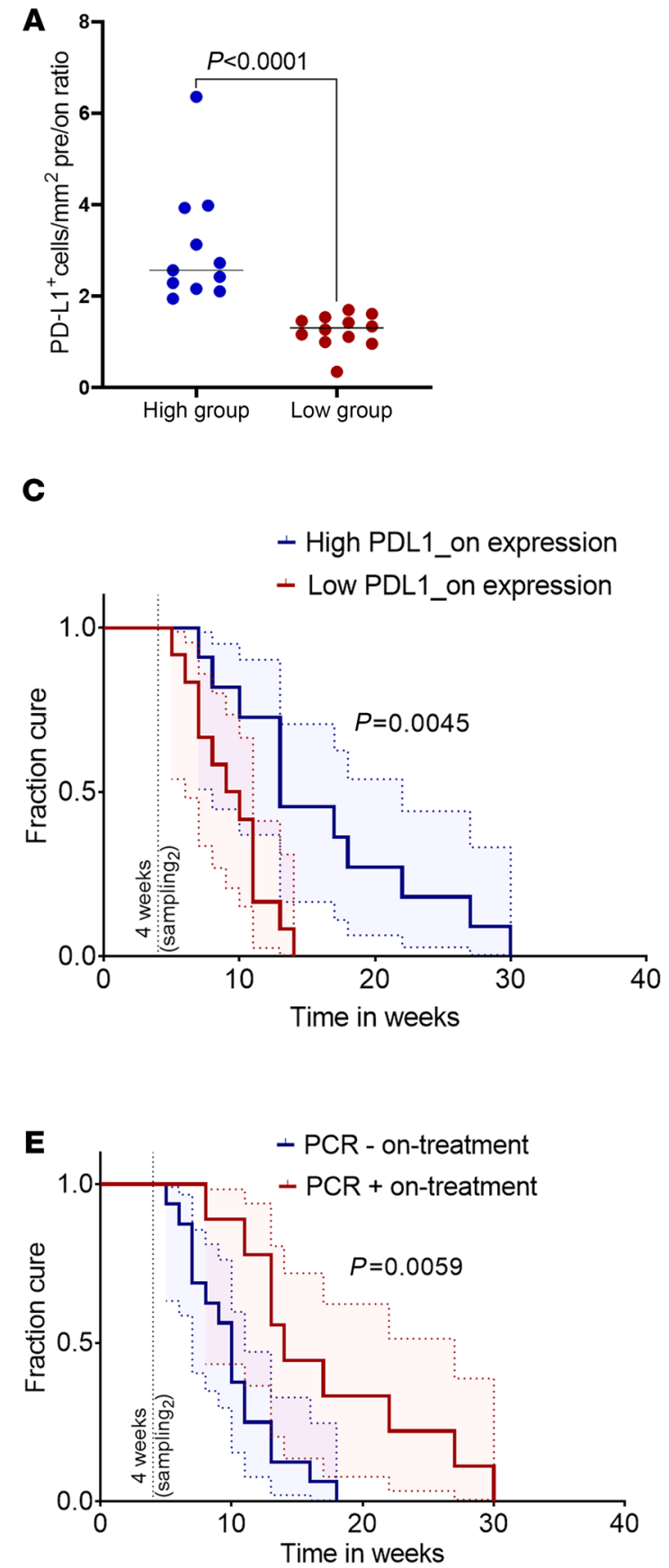
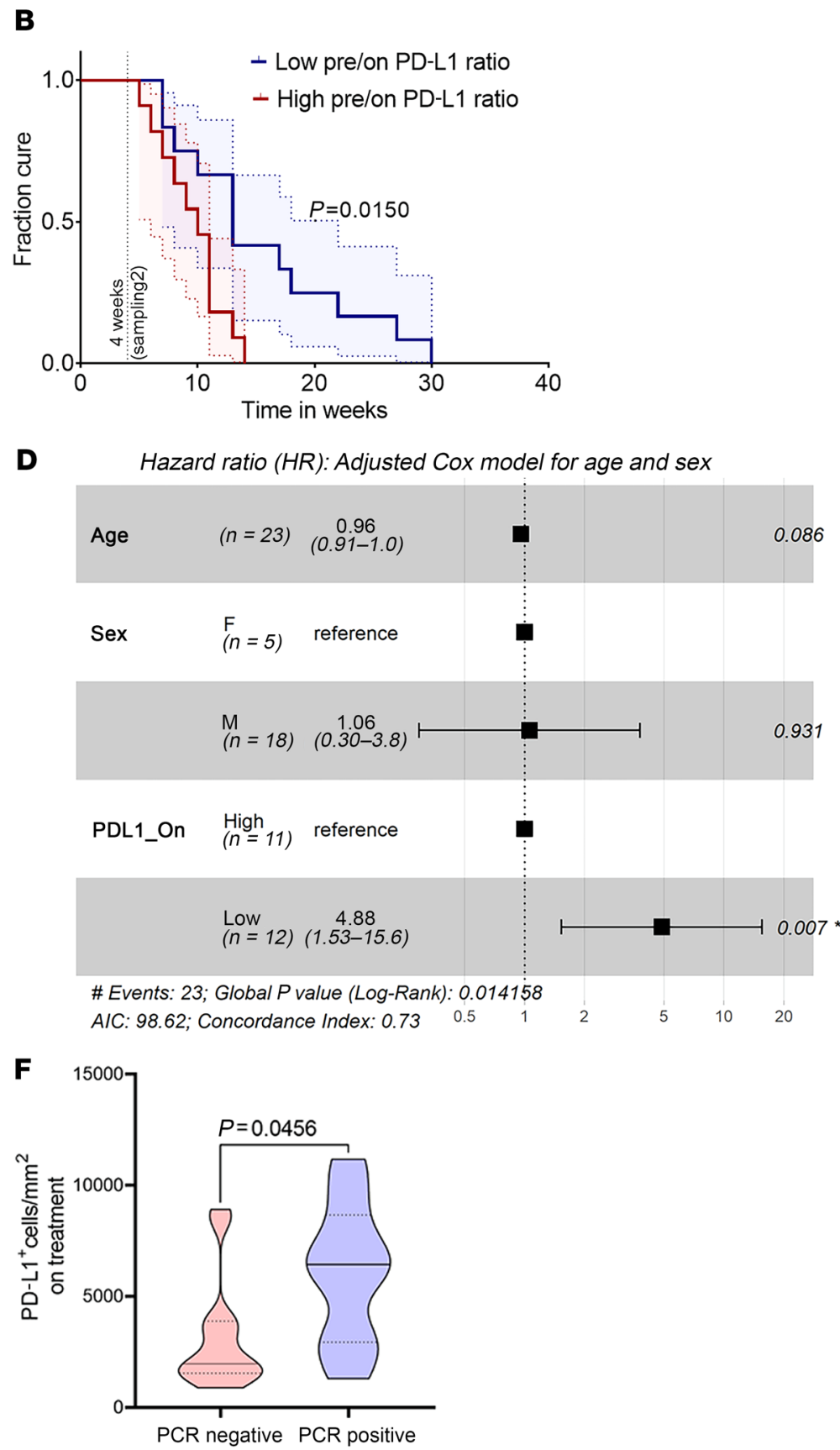

Figure 4. Clinical correlates of PD-L1 reduction on treatment in patients with CL. (A) Patients (validation cohort; $n=23$ ) were stratified based on high (> geomean value; $n=11$ ) and low (< geomean value; $n=12$ ) pre-/on-treatment expression ratio. (B) Kaplan-Meier curve based on pre-/on-treatment ratio of PD-L1 expression (high versus low). (C) Patients stratified based on on-treatment expression of PD-L1 (> geomean value; $n=11$ versus $<$ geomean value; $n=12$ ). (D) Multivariate Cox proportional hazards model plotted as a forest plot. $P$ values for each covariate represent Wald statistic value, and overall statistical significance is also indicated. (E) Patients stratified by LITS1 PCR status ( $n=9 \mathrm{PCR}^{+}$versus $n=14 \mathrm{PCR}^{-}$or PCR ${ }^{+-}$[equivocal]) on treatment. (F) $\mathrm{PD}-\mathrm{L} 1$ expression in LITS PCR ${ }^{+}$versus PCR- individuals on treatment. Dotted lines show upper and lower quantile, solid line shows median. $P$ value generated using 2-tailed Mann-Whitney test. Vertical line drawn in B, C, and E on the $x$ axis shows time when on-treatment biopsies were collected. Curves in B, $\mathbf{C}$, and $\mathbf{E}$ were compared using log-rank (Mantel-Cox) test. Blue and red shaded areas show $95 \% \mathrm{Cl}$ of the 2 groups.

more IDO1 than those with fewer or no Amastin transcripts (Supplemental Figure 12, I-K). These data show that, although a notable population of uninfected $\mathrm{CD} 68^{+}$cells contribute to PD-L1 and IDO1 expression within CL lesions, intracellular parasitism leads to heightened expression of these checkpoint molecules in lesional monocytes and macrophages.
Finally, we tested whether reduction in IDO1 or PD-L1 expression early during therapy could be used as a prognostic marker for treatment response. Patients with the greatest reduction in PD-L1 expression (i.e., greater than the geomean of the pretreatment: on-treatment expression ratio; $n=12$ patients; Figure 4 , A and B) were cured earlier than those who had lower or no reduction in PD-L1 expression $(P=$ 
0.015). Patients with lower PD-L1 expression after 4 weeks of treatment (i.e., lower than the geomean of on-treatment expression; $n=12$ patients) also were cured faster $(P=0.0045$; Figure $4 C)$. We assessed the association of PD-L1 with disease cure rate using univariate Cox proportional hazard regression (Supplemental Figure 13A; HR = 3.96, $P=0.008)$. Upon adjustment for age and sex of the participants, HR increased to $4.88(P=0.007$; Figure $4 \mathrm{D})$, indicating that patients with maximally reduced PD-L1 expression upon treatment were about 5 times more likely to be cured earlier. Conversely, patients remaining parasite $\mathrm{PCR}^{+}$at 4 weeks after treatment had a significantly longer cure time (Figure 4E) and higher PD-L1 expression (Figure 4F). Surprisingly, reduction in IDO1 expression, calculated as either pretreatment/on-treatment expression ratio or IDO1 expression at 4 weeks ( $n=12$ versus $n=11$ ), did not correlate with cure rate (Supplemental Figure 13, B and C). Thus, the relationship between declining PD-L1 expression and rate of cure appears selective.

We conclude that expression of IDO1 and PD-L1 immune checkpoint molecules is a common feature of Sri Lankan CL and that intracellular parasitism is associated with heightened expression of these immunoregulatory proteins in lesional macrophages. Tissue expression of both IDO1 and PD-L1 is markedly reduced within 2 to 4 weeks of treatment onset and well in advance of clinical cure, and a reduction in PD-L1 is associated with a more rapid therapeutic response. The elevated expression of negative immune regulators on macrophages at the lesion site, as demonstrated here, has clear parallels with tumor-associated macrophages (36) and extends our understanding of how Leishmania parasites influence the function of their host cell during human disease (37). Though longitudinal sampling of the same macrophage population was not possible, it seems likely that reduction of PD-L1 expression is facilitated by the leishmanicidal action of SSG, suggesting a model for drug-immune synergy whereby early rounds of SSG treatment reduce intracellular parasite burden, leading to reduced checkpoint inhibition and reengagement of $\mathrm{T}$ cell effector function. Our data, together with strong preclinical evidence of an inhibitory role of PD-L1 in various forms of leishmaniasis $(10,12,38)$, support the candidacy of PD-L1 blockade as an adjunct HDT in Sri Lankan CL. In addition, our data suggest the possibility that changes in PD-L1 expression early after treatment could be considered as a biomarker to trigger drug tapering or drug cessation.

\section{Methods}

A complete, detailed description of Methods is provided in the supplemental material.

Study approval. The study was conducted in accordance with the principles of the Declaration of Helsinki and was approved by the Ethical Review Committee of the Faculty of Medical Sciences, University of Jayewardenepura (ref: 780/13 \& 52/17) and the Department of Biology, University of York. Written informed consent, including for lesion photographs, was received from participants prior to inclusion in this study.

\section{Author contributions}

NSD, SS, VS, NPM, BS, MMDO, LCR, SM, and SR conducted experiments. NSD and SF performed data analysis. NSD and PMK wrote the manuscript. PBW, MC, HG, RW, DL, PMK, and SR were involved in conceptualization and securing funding. PMK and SR supervised the study. The order of the co-first authors was determined by their relative contributions to the study.

\section{Acknowledgments}

The authors thank histopathologist Pushpa Ilanngasinghe (Teaching Hospital Anuradhapura, Sri Lanka), phlebotomist Dawei Chen (University of York, United Kingdom), flow cytometry expert Karen Hogg (University of York, United Kingdom), and technical support at Nanostring Technologies, United States of America, TissueGnostics, Austria, and Centre for Genomic Research, University of Liverpool, United Kingdom. This work was supported by funding from the UK Medical Research Council / UK Aid Global Challenges Research Fund (MR/P024661/1 to PMK, SR, HG, and MC) and a Wellcome Trust Senior Investigator Award (WT104726 to PMK). The funders had no role in the design or conduct of the study or the decision to publish.

Address correspondence to: Paul M. Kaye, Hull York Medical School, University of York, Heslington, York YO10 5DD, United Kingdom. Phone: 44.1904.328840; Email: paul.kaye@york.ac.uk. Or to: Shalindra Ranasinghe, Department of Parasitology, University of Sri Jayewardenepura, Gangodawila, Nugegoda 10250, Sri Lanka. Phone: 94.11.2801028; ishalindra@sjp.ac.lk.
1. Pires M, et al. The impact of leishmaniasis on mental health and psychosocial well-being: a systematic review. PLoS One. 2019;14(10):e0223313.

2. Alvar J, et al. Leishmaniasis worldwide and global estimates of its incidence. PLoS One. 2012;7(5):e35671.

3. Drugs for Neglected Diseases Initiative. Cutaneous leishmaniasis. https://dndi.org/diseases/cutaneousleishmaniasis/. Accessed October 4, 2021.

4. Athukorale DN, et al. Locally acquired cutaneous leishmaniasis in Sri Lanka. J Trop Med Hyg. 1992;95(6):432-433.

5. Karunaweera ND, et al. Sri Lankan cutaneous leishmaniasis is caused by Leishmania donovani zymodeme MON-37. Trans R Soc Trop Med Hyg. 2003;97(4):380-381.

6. Ranasinghe $\mathrm{S}$, et al. Leishmania donovani zymodeme MON-37 isolated from an autochthonous visceral leishmaniasis patient in Sri Lanka. Pathog Glob Health. 2012;106(7):421-424.

7. Zhang WW, et al. Genetic analysis of Leishmania donovani tropism using a naturally attenuated cutaneous strain. PLoS Pathog. 2014;10(7):e1004244.

8. Refai FW, et al. Nonresponsiveness to standard treatment in cutaneous leishmaniasis: a case series from Sri Lanka. Trop Parasitol. 2016;6(2):155-158.

9. Scott P, Novais FO. Cutaneous leishmaniasis: immune responses in protection and pathogenesis. Nat Rev Immunol. 2016;16(9):581-592.

10. da Fonseca-Martins AM, et al. Immunotherapy using anti-PD-1 and anti-PD-L1 in Leishmania amazonensis-infected BALB/c mice reduce parasite load. Sci Rep. 2019;9(1):20275.

11. Brown JA, et al. Blockade of CD86 ameliorates
Leishmania major infection by down-regulating the Th2 response. JInfect Dis. 1996;174(6):1303-1308.

12. Liang SC, et al. PD-L1 and PD-L2 have distinct roles in regulating host immunity to cutaneous leishmaniasis. Eur J Immunol. 2006;36(1):58-64.

13. Greenwald RJ, et al. Cutting edge: inducible costimulator protein regulates both Th1 and Th2 responses to cutaneous leishmaniasis. JImmunol. 2002;168(3):991-995.

14. Mou Z, et al. Parasite-derived arginase influences secondary anti-Leishmania immunity by regulating programmed cell death-1-mediated CD4+ T cell exhaustion. J Immunol. 2013;190(7):3380-3389.

15. Makala LH, et al. Leishmania major attenuates host immunity by stimulating local indoleamine 2,3-dioxygenase expression. J Infect Dis. 2011;203(5):715-725.

16. Okwor I, et al. Deficiency of CD40 reveals an 
important role for LIGHT in anti-leishmania immunity. J Immunol. 2015;195(1):194-202.

17. Akiba $\mathrm{H}$, et al. Critical contribution of OX4O ligand to $\mathrm{T}$ helper cell type 2 differentiation in experimental leishmaniasis. JExp Med. 2000;191(2):375-380.

18. Dalton JE, Kaye PM. Immunomodulators: use in combined therapy against leishmaniasis. Expert Rev Anti Infect Ther. 2010;8(7):739-742.

19. Sbaraglini ML, et al. Neglected tropical protozoan diseases: drug repositioning as a rational option. Curr Top Med Chem. 2016;16(19):2201-2222.

20. Rao SPS, et al. Drug discovery for kinetoplastid diseases: future directions. ACS Infect Dis. 2019;5(2):152-157.

21. Farias Amorim C, et al. Localized skin inflammation during cutaneous leishmaniasis drives a chronic, systemic IFN-gamma signature. PLoS Negl Trop Dis. 2021;15(4):e0009321.

22. Szklarczyk D, et al. STRING v11: protein-protein association networks with increased coverage, supporting functional discovery in genomewide experimental datasets. Nucleic Acids Res. 2019;47(d1):D607-D613.

23. Goncalves R, et al. Platelet activation attracts a subpopulation of effector monocytes to sites of Leishmania major infection. JExp Med. 2011;208(6):1253-1265.

24. Leon B, et al. Monocyte-derived dendritic cells formed at the infection site control the induction of protective $\mathrm{T}$ helper 1 responses against Leishmania. Immunity. 2007;26(4):519-531.

25. Ives A, et al. Leishmania RNA virus controls the severity of mucocutaneous leishmaniasis. Science. 2011;331(6018):775-778.

26. Novais FO, et al. Genomic profiling of human Leishmania braziliensis lesions identifies transcriptional modules associated with cutaneous immunopathology. J Invest Dermatol. 2015;135(1):94-101.

27. Santos Cda S, et al. CD8(+) granzyme B(+)mediated tissue injury vs. CD4(+)IFNgam$\mathrm{ma}(+)$-mediated parasite killing in human cutaneous leishmaniasis. JInvest Dermatol. 2013;133(6):1533-1540.

28. Boussoffara T, et al. Activated cytotoxic T cells within zoonotic cutaneous leishmaniasis lesions. Immun Inflamm Dis. 2019;7(3):95-104.

29. Beechem JM. High-plex spatially resolved RNA and protein detection using digital spatial profiling: a technology designed for immuno-oncology biomarker discovery and translational research. Methods Mol Biol. 2020;2055:563-583.

30. Powles T, et al. MPDL3280A (anti-PD-L1) treatment leads to clinical activity in metastatic bladder cancer. Nature. 2014;515(7528):558-562.

31. Igarashi $\mathrm{T}$, et al. Scoring of PD-L1 expression intensity on pulmonary adenocarcinomas and the correlations with clinicopathological factors. ESMO Open. 2016;1(4):e000083.

32. Roy $S$, et al. Crosstalk of PD-1 signaling with the SIRT1/FOXO-1 axis during the progression of visceral leishmaniasis. JCell Sci. 2019;132(9):jcs226274.

33. Donovan MJ, et al. Indoleamine 2,3-dioxygenase (IDO) induced by Leishmania infection of human dendritic cells. Parasite Immunol. 2012;34(10):464-472.

34. Filippis $C$, et al. Nivolumab enhances in vitro effector functions of PD-1 ${ }^{+} \mathrm{T}$-lymphocytes and Leishmania-infected human myeloid cells in a host cell-dependent manner. Front Immunol. 2017;8:1880.

35. Wang F, et al. RNAscope: a novel in situ RNA analysis platform for formalin-fixed, paraffin-embedded tissues. JMol Diagn. 2012;14(1):22-29.

36. Cassetta L, Pollard JW. Targeting macrophages: therapeutic approaches in cancer. Nat Rev Drug Discov. 2018;17(12):887-904.

37. Kaye P, Scott P. Leishmaniasis: complexity at the host-pathogen interface. Nat Rev Microbiol. 2011;9(8):604-615.

38. Joshi T, et al. B7-H1 blockade increases survival of dysfunctional CD8(+) T cells and confers protection against Leishmania donovani infections. PLoS Pathog. 2009;5(5):e1000431. 